\title{
Inflamabilidade de espécies vegetais do cerrado stricto sensu
}

Francisca de Cássia Silva da Silva ${ }^{a^{*}}$, Victor Braga Rodrigues Duarte ${ }^{a}$, Eduardo Ganassoli Neto ${ }^{a}$, Igor Viana Sousa ${ }^{\mathrm{a}}$, Marcos Vinícius Cardoso da Silva ${ }^{\mathrm{a}}$, Micael Moreira Santos ${ }^{\mathrm{a}}$, Augustus Caeser Frank Portella ${ }^{\mathrm{a}}$, Marcos Giongo ${ }^{\mathrm{a}}$

a Universidade Federal do Tocantins, Brasil

*Autor correspondente (cassia96silva@gmail.com)

\section{N F O}

Keywords

flammability index Brazilian savannah forest fires
Palavras-chaves

índice de

inflamabilidade

Savana brasileira incêndios florestais

\begin{abstract}
A B S T R A C T
Flammability of cerrado stricto sensu plant species.

Fire can cause damage and benefits to an ecosystem, and this relationship is influenced by two main factors: local weather conditions and fuel material characteristics. One of the main factors influencing the combustion process is the flammability of fuels, and its understanding is essential for the prevention of forest fires. Therefore, this study aimed to evaluate the flammability potential of Tabebuia aurea, Pseudobombax grandiflorum, Dimorphandra mollis, Chytraculia concinna, Eriotheca gracilipes and Qualea multiflora of cerrado sensu stricto and to indicate the species with the greatest potential to help prevent forest fires. The investigation was carried out at the Forest Research Laboratory at the Center for Environmental Monitoring and Fire Management (CeMAF) at the Federal University of Tocantins where flammability tests were conducted to evaluate ignition time, ignition frequency, combustion duration, combustion index and flammability value of the combustible materials, for each species studied 50 firings were performed in a epiradiator, each burn consisted of $1 \pm 0.1 \mathrm{~g}$ of green combustible material. Based on the obtained data it was verified that among the studied species the one that showed the highest flammability index was Eriotheca gracilipes, on the other hand, Tabebuia aurea, Pseudobombax grandiflorum and Dimorphandra mollis species are considered to be weakly flammable, classifying them as potential for use in forest fire prevention.
\end{abstract}

\section{R E S U M O}

O uso do fogo pode ocasionar danos e benefícios a um ecossistema, sendo essa relação influenciada por dois fatores principais: condições meteorológicas locais e característica do material combustível. Um dos principais fatores que influenciam o processo de combustão é a inflamabilidade dos combustíveis, sendo considerado essencial a compreensão deste para a prevenção de incêndios florestais. Diante disso este trabalho objetivou avaliar o potencial de inflamabilidade das espécies Tabebuia aurea, Pseudobombax grandiflorum, Dimorphandra mollis, Chytraculia concinna, Eriotheca gracilipes e Qualea multiflora do cerrado sensu stricto e indicar as espécies com maior potencial para auxiliar na prevenção de incêndios florestais. O trabalho foi realizado no Laboratório de Pesquisas Florestais no Centro de Monitoramento Ambiental e Manejo do Fogo (CeMAF) na Universidade Federal do Tocantins onde os testes de inflamabilidade foram conduzidos para avaliação do tempo para ignição, da frequência de ignição, duração da combustão, índice de combustão e valor de inflamabilidade dos materiais combustíveis, sendo que para cada espécie estudada foram efetuadas 50 queimas em epirradiador, cada queima constituída de $1 \pm 0,1 \mathrm{~g}$ de material combustível verde. Com base nos dados obtidos verificou-se que dentre as espécies estudadas a que demonstrou maior índice de inflamabilidade foi a Eriotheca gracilipes, por outro lado, identificouse que as espécies Tabebuia aurea, Pseudobombax grandiflorum e Dimorphandra mollis são consideradas como fracamente inflamáveis, classificando-as como potenciais para uso na prevenção de incêndios florestais. 


\section{INTRODUÇÃO}

O Cerrado é o segundo maior bioma em extensão territorial na América do Sul, ocupa uma área total de $2.036 .448 \mathrm{~km}^{2}$, correspondente a cerca de $22 \%$ do território brasileiro (Brasil, 2017). A vegetação do bioma Cerrado apresenta características que englobam formações florestais, savânicas e campestres (Ribeiro e Walter, 1998).

O Cerrado apresenta vínculo direto com o fogo, tendo em vista que em savanas o uso do fogo é uma estratégia ecológica habitual, podendo atuar como agente de evolução para espécies florestais (Miranda et al., 2008). O fogo desempenha um papel ecológico e social no bioma Cerrado, no entanto a elevada proporção e frequência dos incêndios compromete sua biodiversidade. Diante da relação histórica do Cerrado com o fogo o bioma evoluiu por meio de adaptações que o tornaram, em alguns casos, dependente da ação do fogo (Coutinho, 1990).

$\mathrm{O}$ uso do fogo pode gerar danos e benefícios a um ecossistema, sendo essa relação influenciada por dois fatores principais: condições meteorológicas locais e característica do material combustível (Hernando, 2009). O material combustível é todo o material orgânico presente na floresta e que está passível de ser queimado. A influência desse material no comportamento do fogo e por consequência na ação do fogo sobre o ecossistema irá variar de acordo com suas características, tais como: disponibilidade, distribuição, tipologia, umidade, entre outros (Soares et al., 2017).

Um dos principais fatores que influenciam o processo de combustão é a inflamabilidade dos combustíveis florestais. A inflamabilidade está relacionada à propensão do material combustível em entrar em ignição, as propriedades que mantém o combustível queimando, a velocidade com que o combustível queima e a quantidade de combustível queimado (Hernando, 2009).

De acordo com Batista et al. (2009), existem muitas técnicas e medidas a serem implementadas para impedir ou diminuir os danos ocasionados pelos incêndios, principalmente mediante preparo dos materiais fonte de ignição e prevenção da propagação do incêndio, e conhecer as características da vegetação que compõe a floresta é essencial para a escolha da técnica mais eficaz para determinada área.

Considerando a ausência de pesquisas direcionadas ao tema e que o combustível pode influenciar na efetividade ou suspensão do fogo, tendo em vista que os demais elementos clima, relevo e tempo são classificados como incontroláveis, a compreensão das características de inflamabilidade de espécies do Cerrado pode ser considerada essencial para a prevenção a incêndios florestais.
Diante disso este trabalho objetivou avaliar o potencial de inflamabilidade das espécies Tabebuia aurea, Pseudobombax grandiflorum, Dimorphandra mollis, Chytraculia concinna, Eriotheca gracilipes e Qualea multiflora, de grande ocorrência no cerrado sensu stricto, e indicar as espécies com maior potencial para auxiliar na prevenção de incêndios florestais.

\section{MATERIAL E MÉTODOS}

O trabalho foi realizado no Laboratório de Pesquisas Florestais no Centro de Monitoramento Ambiental e Manejo do Fogo (CeMAF) na Universidade Federal do Tocantins, campus de Gurupi, sob as respectivas coordenadas geográficas $11^{\circ} 46^{\prime} 25^{\prime \prime}$ $\mathrm{S}$ e $49^{\circ} 02^{\prime} 05^{\prime \prime} \mathrm{W}$.

Foram coletados folhas e ramos das espécies $T a$ bebuia aurea (Silva Manso) Benth. \& Hook.f. ex S.Moore (craíba), Pseudobombax grandiflorum (Cav.) A. Robyns (embiruçu), Dimorphandra mollis Benth (faveira), Chytraculia concinna (DC.) Kuntz (guamirim), Eriotheca gracilipes (K. Schum.) A. Robyns (paineira-do-campo) e Qualea multiflora Mart. (pau-terra-liso) com diâmetro inferior a $0,7 \mathrm{~cm}$, classificados como material combustível fino (Soares et al., 2017).

Para determinar o teor de umidade do material combustível foi utilizada a equação 1 (Batista, 1990), sendo a massa seca obtida através de $200 \mathrm{~g}$ das amostras secas levadas à estufa por 48 horas a $75 \mathrm{C}$.

$$
U=\left(\frac{M U-M S}{M S}\right) * 100
$$

$$
\begin{array}{cl}
\text { Onde: } & \\
\mathrm{U} & \text { teor de umidade do material }(\%) \\
\mathrm{MU} & \begin{array}{l}
\text { massa úmida do material no momento } \\
\text { da coleta }(\mathrm{g}) ;
\end{array} \\
\mathrm{MS} & \text { massa seca do material após estufa }(\mathrm{g}) .
\end{array}
$$

O teste de inflamabilidade foi conduzido conforme a metodologia proposta por Valette (1992), Petriccione (2006) e Kovalsyki et al. (2016). O material combustível foi submetido às avaliações até o tempo máximo de 2 horas após sua coleta em campo, isso se deve em razão da necessidade de manter as condições das amostras as mais naturais possíveis (Kovalsyki, 2016). O processo de queima do material combustível foi realizado em epirradiador em condições controladas, sem interferência de correntes de ar para assegurar a homogeneidade das condições para todas a queimas. Foi alocado, 4 $\mathrm{cm}$ acima do epirradiador, uma chama piloto que tem como objetivo auxiliar na combustão inicial (Figura 01). 


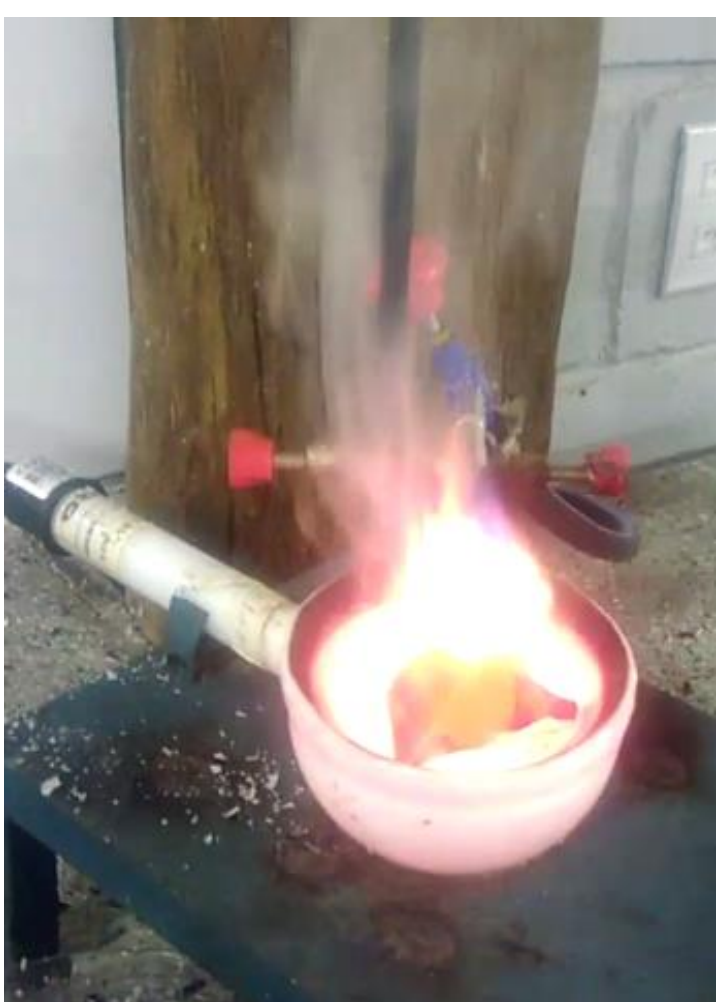

Figura 01 - Epirradiador durante a queima do material combustível.
Para cada espécie estudada, foram efetuadas 50 queimas. Cada queima foi constituída de $1 \pm 0,1 \mathrm{~g}$ de material combustível verde, obtido através de uma balança de precisão. Todas os passos para a realização da queima foram executados sem entrar em contado direto com o material, com a utilização de luvas de látex, para evitar influências em suas propriedades. As características da combustão analisadas, de acordo com o proposto por Petriccione (2006), foram:

- tempo para ignição (TI): tempo percorrido até o início da combustão do material combustível;

- frequência de ignição (FI): número de vezes em que ocorreu ignição, considerando um tempo de ignição de 60 segundos, sendo que queimas que ultrapassaram este tempo foram consideradas como "queimas negativas";

- duração da combustão (DC): duração do tempo em que a chama permaneceu acesa;

- índice de combustão (IC): energia de combustão da queima, definida por meio da média do comprimento da chama e classificada segundo os índices dispostos na Tabela 01.

- valor de inflamabilidade (VI): classificado mediante o FI e o TI (Tabela 02).

Tabela 01 - Índices do valor de combustão.

\begin{tabular}{ccc}
\hline Índice de combustão (IC) & Designação do IC & Comprimento da chama (cm) \\
\hline ICI & Muito baixa & $<1$ \\
IC2 & Baixa & 1 a 3 \\
IC3 & Média & 4 a 7 \\
IC4 & Alta & 8 a 12 \\
IC5 & Muito alta & $>12$ \\
\hline
\end{tabular}

Fonte: Petriccione (2006).

Tabela 02 - Índices do valor de inflamabilidade.

\begin{tabular}{ccccccc}
\hline \multirow{2}{*}{ TI (s) } & \multicolumn{7}{c}{ FI } & & & \\
\cline { 2 - 7 } & $<25$ & $\mathbf{2 5 - 3 8}$ & $\mathbf{3 9 - 4 1}$ & $\mathbf{4 2 - 4 4}$ & $\mathbf{4 5 - 4 7}$ & $\mathbf{4 8 - 5 0}$ \\
\hline$>32,5$ & 0 & 0 & 0 & 1 & 1 & 2 \\
$27,6-32,5$ & 0 & 0 & 1 & 1 & 2 & 2 \\
$22,6-27,5$ & 0 & 0 & 1 & 2 & 2 & 2 \\
$17,6-22,5$ & 1 & 1 & 2 & 2 & 3 & 3 \\
$12,6-17,5$ & 1 & 1 & 2 & 3 & 3 & 4 \\
$<12,6$ & 1 & 2 & 3 & 3 & 4 & 5 \\
\hline
\end{tabular}

Nota: FI - frequência de ignição e TI - tempo para ignição (Fonte: Petriccione, 2006).

$\mathrm{O}$ valor de inflamabilidade foi identificado através do número correspondente entre o tempo de ignição e a frequência de ignição, classificado em: VI $=0$ (fracamente inflamável); $\mathrm{VI}=1$ (pouco inflamável); VI = 2 (moderadamente inflamável); VI = 3 (inflamável); VI = 4 (altamente inflamável) ou VI $=5$ (extremamente inflamável) (Valette, 1992).

\section{RESULTADOS E DISCUSSÃO}

Na tabela 03 podem ser observados os resultados das queimas das espécies, seus percentuais de umidade e a frequência com que sofreram ignição. Nota-se que para os parâmetros da combustão a espécie que apresentou os melhores resultados foi a 
$P$. grandiflorum com alto tempo médio para ignição $(60,83 \mathrm{~s})$, baixa duração média de queima $(0,42 \mathrm{~s})$ e baixa altura média de chama $(0,16 \mathrm{~cm})$.

A espécie E. gracilipes apresentou queimas com altas intensidades, com baixo tempo médio para ignição (17,62 s) e altura média de chama elevada $(16,42 \mathrm{~cm})$. As variáveis que influenciam no comportamento do fogo, sobretudo o tempo para ignição e o comprimento inicial da chama, estão associadas ao teor de umidade dos materiais combustíveis (Hernando, 2009). Essa afirmação pode ser observada na espécie mencionada que apresentou baixo teor de umidade, porém a espécie $C$. concinna apresentou alto teor de umidade e parâmetros da queima elevados, necessitando de um estudo mais aprofundado sobre a espécie.

Kovalsyki et al. (2016) avaliando espécies para compor cortinas de segurança utilizou a espécie $P i$ nus taeda como controle e observou para a espécie um TI de $18,4 \mathrm{~s}$, uma DC de $11,8 \mathrm{~s}$ e uma HC de $12,1 \mathrm{~cm}$ a uma umidade de $136 \%$ do material. Ao compararmos os resultados do autor supracitado aos obtidos neste trabalho para as espécies com umidades próximas, $P$. grandiflorum e $D$. mollis, observamos que os TI obtidos para essas espécies são muito superiores, 60,83 e 45,58 s, respectivamente, enquanto que os valores de DC $(0,42$ e 2,69 s) e $\mathrm{HC}(0,16$ e $3,96 \mathrm{~cm})$ são muito inferiores, os menores dentre as espécies avaliadas. Estes resultados indicam que tais espécies possuem potencial para compor cortinas de segurança em plantios comerciais de P. taeda.

Tabela 03 - Resultados do teste de inflamabilidade para espécies estudadas.

\begin{tabular}{|c|c|c|c|c|c|}
\hline \multirow{2}{*}{ Espécies } & \multicolumn{3}{|c|}{ Médias } & \multirow{2}{*}{$\mathbf{U} \% *$} & \multirow{2}{*}{$\mathbf{F I}^{*}$} \\
\hline & TI $(s) *$ & $\mathrm{DC}(\mathrm{s})^{*}$ & $\mathrm{HC}(\mathrm{cm})^{*}$ & & \\
\hline E. gracilipes & 17,62 & 16,55 & 16,42 & 52,38 & 2 \\
\hline T. aurea & 48,20 & 7,70 & 2,76 & 54,3 & 0 \\
\hline P. grandiflorum & 60,83 & 0,42 & 0,16 & 111,38 & 2 \\
\hline D. mollis & 45,58 & 2,69 & 3,96 & 103,39 & 40 \\
\hline C. concinna & 13,41 & 4,11 & 15,17 & 306,99 & 19 \\
\hline Q. multiflora & 21,13 & 7,83 & 16,16 & 217,17 & 27 \\
\hline
\end{tabular}

TI (s) - tempo para ignição; DC (s) - duração da combustão; HC (cm) - altura da chama; U\% - umidade do material; FI - frequência de ignição.

Observando a tabela 04 verifica-se que as espécies $T$. aurea, $P$. grandiflorum e D. mollis obtiveram menores valores de índice de combustão, 2, 1 e 3 , respectivamente, e o valor de inflamabilidade foi igual a 0 (fracamente inflamável), resultando assim nas espécies menos inflamáveis. Esse resultado pode estar relacionado com as variáveis tempo de ignição e altura de chama, pois para essas espécies obtiveram-se os menores valores de altura de chama e os mais longos tempos para ignição.

Tabela 04 - Índice de combustão e valor de inflamabilidade de seis espécies do Cerrado.

\begin{tabular}{lcc}
\hline \multicolumn{1}{c}{ Espécies } & IC & VI \\
\hline E. gracilipes & 5 & 3 \\
T. aurea & 2 & 0 \\
$P$. grandiflorum & 1 & 0 \\
D. mollis & 3 & 0 \\
C. concinna & 5 & 1 \\
Q. multiflora & 5 & 1 \\
\hline
\end{tabular}

As espécies $C$. concinna e $Q$. multiflora apresentaram resultado para valor de inflamabilidade igual a 1 (pouco inflamável), caracterizando-as como espécies com inflamabilidade muito baixa, devido ao tempo para ignição maior, e a altura de chama com um valor elevado em relação às espécies que obtiveram a classificação do índice de inflamabilidade 0 . Essas espécies apresentaram o maior teor de umidade dos materiais coletados, 306,99 e 217,17\%, respectivamente.

Geralmente a umidade foliar chega a $300 \%$ no período de folhação do vegetal e decresce para cerca de $50 \%$ devido ao processo de senescência foliar, e, juntamente aos óleos essenciais voláteis, a umidade dificulta a absorção de energia pelo material durante o processo de combustão, influenciando diretamente na inflamabilidade (Soares \& Batista, 2007; White e Zipperer, 2010).

A espécie com a maior inflamabilidade foi a $E$. gracilipes com a classificação do índice de inflamabilidade igual a 3, caracterizando-a como inflamável, devido ao tempo para ignição ser baixo, a umidade baixa em relação às outras espécies, e o principal fator que contribuiu para esse índice de inflamabilidade alto: o tempo que a chama se manteve acesa. Batista et al. (2009) avaliando a inflamabili- 
dade de Ligustrum lucidum, espécie largamente utilizada na arborização urbana, identificou uma grande influência do teor de umidade do material nos parâmetros da combustão.

Em levantamento de plantas de baixa inflamabilidade Souza e Vale (2019) observaram baixa inflamabilidade das espécies Vochysia thyrsoidea, Palicourea rigida, e Lavoisiera bergii no cerrado do Distrito Federal, indicando-as para serem utilizadas em aceiros verdes. Kovalsyki et al. (2016) investigaram espécies para compor cortinas de segurança e observaram que as espécies Bougainvillea glabra e Schinus terebinthifolius foram as melhores, ambas com IC $=2$ e VI $=0$, e ressaltaram esse resultado ao compará-las a espécie Pinus taeda que se mostrou inflamável de acordo com os testes.

Realizando uma comparação com os dados obtidos neste estudo verificou-se que as espécies $T$. $a u$ rea, $P$. grandiflorum e $D$. mollis ficaram com valores próximos as espécies com baixas inflamabilidades apontadas pelos trabalhos mencionados, isso se deve a vários fatores como, por exemplo, umidade do material, a sua composição química e espessura da folha.

\section{CONCLUSÃO}

Com base nos resultados deste estudo podemos verificar que dentre as espécies estudadas a que demonstrou maior índice de inflamabilidade foi a $E$. gracilipes. Por outro lado, identificou-se que as espécies $T$. aurea, $P$. grandiflorum e $D$. mollis são consideradas como fracamente inflamáveis, classificando-as como potenciais para uso na prevenção de incêndios florestais.

\section{REFERÊNCIAS BIBLIOGRÁFICAS}

BATISTA, A.C. Incêndios florestais. Recife: Imprensa Universitária da UFRPE, 115 p. 1990.

BATISTA, A.C.; BIONDI, D. Avaliação da inflamabilidade de Ligustrum licidum Aiton (Oleaceae) para uso potencial em cortinas de segurança na região sul do Brasil. Revista Brasileira de Ciências Agrárias, v. 4, n. 4, p. 435-439, 2009. https://doi.org/10.5039/agraria.v4i4a11

BRASIL. O Bioma Cerrado. Ministério do Meio Ambiente (MMA). 2018. Disponível em: <

http://www.mma.gov.br/biomas/cerrado>, acesso em: 21 de abril de 2019.

COUTINHO, L.M. O cerrado e a ecologia do fogo. Ciência hoje, v. 12, n. 68, p. 23-30, 1990.

HERNANDO, C. Combustibles forestales: inflamabilidad. La defensa contra incendios forestales, fundamentos y experiencias. McGraw-Hill, p. 3-6, 2000.
HERNANDO, C.L. Combustibles forestales: inflamabilidad. In: Vélez, R.M. (Coord). La defensa contra incêndios forestales: fundamentos y experiências, 2. ed. Espanha: Mcgrawhill, 2009.

KOVALSYKI, B.; TAKASHINA, I.C.; TRES, A.; TETTO, A.F.; BATISTA, A.C. Inflamabilidade de espécies arbóreas para uso em cortinas de segurança na prevenção de incêndios florestais. Pesquisa Florestal Brasileira, v.36, n.88, p.387-391, 2016.

https://doi.org/10.4336/2016.pfb.36.88.991

MIRANDA, H.S., BUSTAMANTE, M.M.C.; MIRANDA, A.C. 2002. The fire factor. In Oliveira, PS. and Marquis, RJ. (Eds.). The Cerrados of Brazil: ecology and natural history of a neotropical savanna. New York: Columbia University Press. p.51-68.

NEVES, P.C. Avaliação da inflamabilidade de cinco espécies da floresta ombrófila mista. Universidade Federal do $\mathrm{Pa}$ raná, 2016.

OLIVEIRA, P.S.; MARQUIS, R.J. The cerrados of Brazil: ecology and natural history of a neotropical savanna. Columbia University Press, 2002.

PETRICCIONE, M. Infiammabilità della lettiera di diverse specie vegetali di ambiente mediterraneo. 2006. $48 \mathrm{f}$. Tese (Doutorado em Biologia Aplicada) - Dipartimento di Biologia Strutturale e Funzionale, Università Degli Studi Di Napoli Federico II, Napoli. 2006.

RIBEIRO, J.F.; WALTER, B.M.T. Fitofisionomias do bioma Cerrado. Embrapa Cerrados-Capítulo em livro científico (ALICE). p. 89-166, 1998.

SCARFF, F.R.; WESTOBY, M. Leaf litter flammability in some semi-arid Australian woodlands. Functional Ecology, v. 20, n. 5 , p. 745-752, 2006. https://doi.org/10.1111/j.1365-2435.2006.01174.x

SEPLAN/TO. Atlas do Tocantins: Subsídios ao planejamento da gestão territorial. 2002.

SOARES, R.V.; BATISTA, A.C.; TETTO, A.F. Incêndios florestais: controle, efeitos e uso do fogo. Curitiba, $2^{\mathrm{a}}$ edição revisada, 255p. 2017.

SOUZA, M.A.; VALE, A.T. Levantamento de plantas de baixa inflamabilidade em áreas queimadas de cerrado no Distrito Federal e análise das suas propriedades físicas. Universidade de Brasília, 2019.

VALETTE, J.C. Inflammabilities of Mediterranean species. Forest fire risk and management, v.16719, p.51-64, 1997.

WHITE, R.H.; ZIPPERER, W.C. Testing and classification of individual plants for fire behaviour: plant selection for the wildland-urban interface. International Journal of Wildland Fire, v.19, n.2, p.213-227, 2010. https://doi.org/10.1071/WF07128 